\title{
Transparency Optimized Interaction in Telesurgery Devices via Time-Delayed Communications
}

\author{
Amin Mahdizadeh* \\ IEEE member \\ Technische Universität München
}

\author{
M. Ali Nasseri ${ }^{\dagger}$ and Alois Knoll \\ Department of Robotics and Embedded Systems \\ Institut für Informatik \\ Technische Universität München
}

\begin{abstract}
Low transparency is one of the major drawbacks of passivity-based control approaches in teleoperation systems, particularly when time delay exists in the communication channel. In this work, we improved the transparency of time-delayed teleoperation. For this, we used a passivity-based control approach with the generalized scattering transformation. To maximize a transparency criterion, the parameters of the generalized scattering transformation were optimized. Moreover, we show that communication channel disturbance attenuation is also improved. The proposed approach is validated on a master-slave robot interacting with an human eyeball model via a time delayed communication. Two classes of parameter sets are considered and compared against each other in sense of transparency and stability. Results verify a noticeable enhancement of transparency in stiffer environments by the proposed method.
\end{abstract}

Index Terms: Teleoperation systems, Tele-Robotic ophthalmic surgery, Generalized scattering, Transparency, Optimization.

\section{INTRODUCTION}

Teleoperation systems enable human operators to interact with environments which are physically distant, inaccessible or even dangerous. Relevant applications range from hazardous space missions to the scaled environments like biomedical manipulation and telesurgery. Conventionally, a combination of visual, auditory and haptic information is exchanged between the Tele-Operator (TO) and the Human-System-Interface (HSI) device, to provide a feeling of presence for the user, see Fig.1. In many applications, haptic and force feedback information, help the user to significantly improve the operation and performance of more precise and complex tasks [1]. In such a system setup, the teleoperator interacts with the target environment based on the human operator commands. Depending on the system architecture, the pose, velocity and/or force is fed back to HSI. In an ideal teleoperation system, the human operator has the feeling of natural and direct interaction with the remote environment. A transparent teleoperation system is a system which, the user does not feel dynamics of the intermediate system, i.e. the mechanical impedance displayed to the user equals to the mechanical impedance of the remote environment [2]. However, achieving a high level of transparency is difficult if the communication between HSI and teleoperator suffers from time delay.

Even a small time delay in the communication may result in instability of the teleoperation system, as a global control loop is closed via the communication channel [3]. Also in most of the applications, this time delay value is unknown or varying. The scattering transformation (alternatively called wave variable transfor-

\footnotetext{
*e-mail: a.m.mahdizadehh@ieee.org

†e-mail:nasseri@in.tum.de

‡email:knoll@in.tum.de
}

IEEE Haptics Symposium 2014

23-26 February, Houston, Tx, USA

978-1-4799-3131-6/14/\$31.00 @2014 European Union

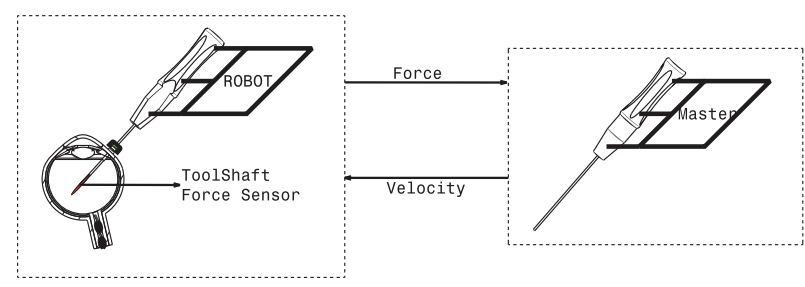

Figure 1: Teleoperation system with multiple types of information exchange used in eye telesurgery

mation) $[4,5]$ is one of the well-known approaches to guarantee stability of teleoperation systems with arbitrarily large time delay based on passivity arguments.

However, it compromises the performance of the system which is conventionally measured as terms of transparency [6]. It means that the impedance which is appeared to the user on the HSI, is not exactly what the environment is. Mathematically speaking, transparency is achieved if the displayed impedance equals to the environment impedance [2]. By having more information about the environment dynamics, a teleoperation system can be improved in sense of stability and transparency [7]. In [8,9], by exploiting the dissipativity parameters of the environment, a more generalized form of the scattering transformation is introduced. The generalized scattering transformation provides adjustable parameters to improve transparency based on the lack or excess of passivity of the individual components of the teleopration system [10]. However, an investigation for finding the optimal parameters in has not been performed yet.

The contribution of this paper is to improve the transparency of the system based on optimal parameter design. The main idea is to introduce a broader class of parameter sets for the generalized scattering and to optimize the system with those parameters as optimization variables under stability constraints. We use a quantitative measure for this similarity to define a cost function which can be used in a suitable optimization tool. Determining parameters of the generalized scattering transformation is a design problem and in this work, the search for the optimal parameters is carried out by using swarm optimization. The preliminary evaluation of the proposed approach for robotic eye surgery [11] considering the mechanical tissue characteristics of the human eye was performed. Master-Slave robot assisted eye surgery is one of the teleoperation areas which is affected the most from the system transparency. Fragility of the anatomy and the required precision for performing ophthalmic procedures, specifically in intraocular operations, are the most important reasons which makes the factor of transparency a must for ophthalmic operation setup.

The remainder of the paper is organized as follows: in section 2, a brief background is given on dissipative systems and generalized scattering. Section 3 derives some desirable properties of generalized scattering transformation parameters. A transparency measure and optimization objective are defined in section 4 . In section 5 and 6 , simulation and experimental results are represented for comparison. 


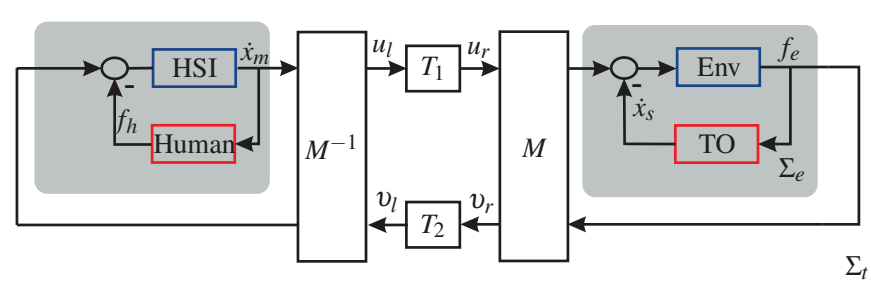

Figure 2: Teleoperation system block diagram with time delay and generalized scattering transformation blocks (marked by $M$ and $M^{-1}$ ).

\section{BACKGROUND}

\subsection{IF-OFP system model for the teleoperation}

In the velocity/force architecture, the environment is considered as a mapping from velocity to force as output. This mapping can be assumed as admittance/impedance models with unknown values of Inertia, damping and stiffness. By having the prior knowledge only about the lower bound for damping from the unknown environment, it can be represented as input-feedforward-output-feedback passive (IF-OFP) systems [12]. This means that the environment is assumed an energy dissipative system with its dissipation rate is bounded from below. Note that also the sub-systems human, HSI, and teleoperator can each be represented by an IF-OFP system. The overall system is a interconnection of different IF-OFP systems and time delays [13]. Consider the system $\Sigma: u \longmapsto y$, where $u \in \mathbb{R}_{p}$ and $y \in \mathbb{R}_{q}$ and $u, y$ are system's input/output respectively. It is called Output Feedback Passive if there exist a positive semi-definite storage function $S: \mathbb{R}_{n} \rightarrow \mathbb{R}_{+}$such that for each admissible $u$ and $t \geq 0$

$$
S(x(t))-S(x(0)) \leq \int_{0}^{t} u^{T} y-\delta u^{T} u-\varepsilon y^{T} y \mathrm{~d} \tau,
$$

for some $\delta, \varepsilon>0$. In a matrix format it can be written as

$$
S(x(t))-S(x(0)) \leq \int_{0}^{t}\left[\begin{array}{ll}
\mathrm{u} & \mathrm{y}
\end{array}\right] \mathbf{P}\left[\begin{array}{l}
\mathrm{u} \\
\mathrm{y}
\end{array}\right] \mathrm{d} \tau,
$$

and

$$
\mathbf{P}=\left[\begin{array}{cc}
\mathbf{Q} & \mathbf{S} \\
\mathbf{S}^{T} & \mathbf{R}
\end{array}\right]
$$

where $\mathbf{Q} \in \mathbb{R}_{p \times p}, \mathbf{R} \in \mathbb{R}_{q \times q}$ and $\mathbf{S} \in \mathbb{R}_{p \times q}$ and $\mathbf{u}, \mathbf{y}$ have dependency on time, $\tau$. Also $p$ and $q$ are number of input and outputs of the system respectively. The matrix $\mathbf{P}$ is also not unique for a system and every set of $\mathbf{Q}, \mathbf{S}$ and $\mathbf{R}$ which satisfies the inequality (2.1) can be acceptable [13]. As a physical description for the above definition, it can be mentioned to mechanical systems which are stimulated by an external energy source. A part of this energy is stored in the system like potential energy in the masses or springs. The other part is dissipated e.g. in terms of heat energy. So, according to the definition, the stored energy of a passive mechanical system, can be only increased by external sources and it does not generate energy by itself. In other word, its stored energy is always equal or less than the energy which is given to the system.

A single-input single-output IF-OFP system can be achieved by choosing $\mathbf{S}=\frac{1}{2}, \mathbf{Q}=-\sigma$ and $\mathbf{R}=-\delta$ which gives

$$
\mathbf{P}=\left[\begin{array}{cc}
-\delta & \frac{1}{2} \\
\frac{1}{2} & -\varepsilon
\end{array}\right],
$$

where $\delta, \varepsilon \in \mathbb{R}$. For $\delta=\varepsilon=0$, passive systems are recovered with the bilinear supply rate $u^{T} y$. The stability of two interconnected IFOFP systems can be guaranteed based on a condition on the their IF-OFP parameters. For this, onsider two IF-OFP systems $\Sigma_{1}$ and $\Sigma_{2}$ with $\delta_{i}, \varepsilon_{i}, i \in 1,2$. The negative feedback interconnection of $\Sigma_{1}$ and $\Sigma_{2}$ is finite gain $\mathscr{L}_{2}$-stable if

$$
\varepsilon_{2}+\delta_{1}>0 \text { and } \varepsilon_{1}+\delta_{2}>0
$$

see also [14] for more details.

This very useful property of IF-OFP systems is used in [8] to derive the parameters of the generalized scattering the to guarantee the delay independent stability of the teleoperation system. The approach will be described in the next section.

\subsection{Generalized scattering}

In order to overcome the destabilizing effects of time delay in the control loop, the generalized scattering transformation is developed in [8] as an extended version of standard scattering transformation [5]. A schematic representation of passivity based teleoperation system with generalized scattering blocks (marked by $\mathbf{M}$ and $\mathbf{M}^{-1}$ ) is shown in Fig.2. In our work we consider the velocityforce architecture. At the right hand side, are the blocks for the teleoperator and environment and at the left hand side the blocks for HSI and human are located. The blocks $M$ and $M^{-1}$ represent the right- and left-hand-side generalized scattering transformation. The communication channel is modeled by a time $T_{1}$ in the forward path and a time delay $T_{2}$ in the backward path. After applying the transformation, $u_{l}$ is transmitted instead of the HSI velocity $\dot{x}_{m}$, and the variable $v_{r}$ is transmitted from the teleoperator side instead of the force. The desired teleoperator velocity $\dot{x}_{s}$ and the desired displayed force $f_{m}$ at the HSI are computed from the transformation equations according to

$$
\left[\begin{array}{l}
u_{l} \\
v_{l}
\end{array}\right]=\mathbf{M}\left[\begin{array}{c}
\dot{x}_{m} \\
f_{m}
\end{array}\right]
$$

and for the left hand side

$$
\left[\begin{array}{c}
\dot{x}_{s} \\
f_{e}
\end{array}\right]=\mathbf{M}^{-1}\left[\begin{array}{c}
u_{r} \\
v_{r}
\end{array}\right] .
$$

Since these transformations are inversion of each other and placed at input/output ports of the subsystems, in case of zero time delay, they cancel each other out. For the right hand side, variables $u_{r}$ and $v_{l}$ denote the output of the time delay blocks in the forward and backward channel, respectively.

The transformation $\mathbf{M}$ is decomposed to a rotation matrix $\mathbf{R}$ and a positive definite matrix $\mathbf{B}$

$$
\mathbf{M}=\mathbf{R} \cdot \mathbf{B}=\left[\begin{array}{cc}
\cos \theta & \sin \theta \\
-\sin \theta & \cos \theta
\end{array}\right]\left[\begin{array}{ll}
b_{11} & b_{12} \\
b_{21} & b_{22}
\end{array}\right],
$$

where $\operatorname{Det}(\mathbf{B}) \neq 0$ and $\theta$ is the rotation angle which is chosen based on the IFP- and OFP-properties of the environment. It can be described how to calculate this angle to guarantee delay-independent stability of the system. The basic idea is to modify the IF-OFP parameters of the block $\Sigma_{t}$ in Fig. 2 such that they become the same as the block $\Sigma_{e}$. If the system is stable then according to the stability criterion for interconnected IF-OFP systems without time delay, it would be also with time delay. In the following a constructive way is presented to compute the transformation parameters achieving this independent of the time delay [9]. For that, consider the subsystem $\Sigma_{t}: \dot{x} \mapsto f$ comprising the IF-OFP environment and teleoperator $\Sigma_{e}$ with the total dissipativity parameters of $\mathbf{Q}_{e}=-\delta_{e}, \mathbf{R}_{e}=\varepsilon_{e}, \mathbf{S}_{e}=\frac{1}{2}$, the static system $\mathbf{M}=\mathbf{R B}$. Time delay $T_{1,2}$ is constant. If for each $\mathbf{B}$ the angle $\theta$ is chosen as one of two solutions of

$$
\cot 2 \theta=\frac{\varepsilon_{B}-\delta_{B}}{2 \eta_{B}}
$$

where $\varepsilon_{B}, \delta_{B}, \eta_{B}$ are the dissipativity parameters of the system $\Sigma_{B}$ which is defined by

$$
\mathbf{P}_{B}=\mathbf{B}^{-T} \mathbf{P}_{e} \mathbf{B}^{-1}
$$




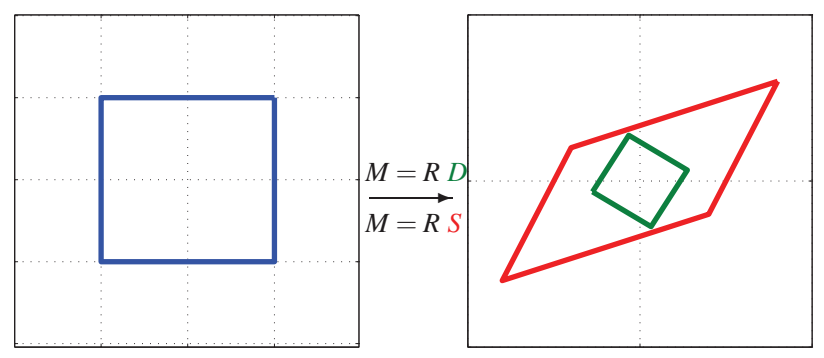

Figure 3: An example of mapping on a set of points. Original shape is square(left). Since $M$ is a right polar decomposition, it is angle preserving when $B$ is diagonal (right-green). With a symmetric $B$ angles can be also skewed (right-red).

Then, the exact environment dissipativity property $\mathbf{P}_{e}$ is preserved into the subsystem $\Sigma_{t}[8]$

$$
\mathbf{P}_{t}=\left[\begin{array}{cc}
-\delta & \frac{1}{2} \\
\frac{1}{2} & -\varepsilon
\end{array}\right]=\mathbf{P}_{e}
$$

Those computed transformation parameters achieve IF-OFP properties for sub-system $\Sigma_{t}$ as for sub-system $\Sigma_{e}$. For stability, however, this equality is not necessary and there is a range of parameters guaranteeing stability [reference Iason, where he provides the angles]. Even though, transparency of the system can be affected if the parameters are badly chosen for a given system. To improve transparency, a matrix $\mathbf{M}$ should be found, which gives the maximum similarity between environment and displayed impedance. In Fig.2, environment and displayed impedance are shown by $\Sigma_{e}$ and $\Sigma_{t}$ respectively. Possibly, within this range of parameters we may find an $\mathbf{M}$ which provides better transparency than the one computed by this method. We will investigate the influence of the generalized scattering transformation parameters on the transparency and the disturbance rejection in the next section.

\section{EfFECTS OF M ON DISPLAYED IMPEDANCE}

On previous works, for simplicity matrix $\mathbf{B}$ is assigned a diagonal matrix for simplification of calculations. However it can be shown that by using a full symmetric matrix we can have a larger class for choosing $\mathbf{B}$, which can improve the transparency. In (3), $\mathbf{M}=\mathbf{R} \mathbf{B}$ is a right polar decomposition of $\mathbf{M}$ where $\mathbf{R} \in S O(2)$ and $\mathbf{B}$ is either a positive semi-definite symmetric matrix $\mathbf{S}$ or a diagonal matrix D. Every right polar decomposition can be written as a left polar decomposition. For example if $\mathbf{A}=\mathbf{U P}$ is the right polar decomposition(RPD) of $\mathbf{A}$, where $\mathbf{U}$ is an orthonormal and $\mathbf{P}$ is a positive symmetric matrix, then it can be alternatively written as a left polar decomposition (LPD) [15]. It can be written as $\mathbf{A}=$ $\mathbf{P}^{\prime} \mathbf{U}$, where $\mathbf{P}^{\prime}=\mathbf{U P U}^{T}$, which is a representation of eigenvalue decomposition of a symmetric matrix.

As $\mathbf{M}=\mathbf{R} \mathbf{B}$ is a right polar decomposition, $\mathbf{M}=\mathbf{R} \mathbf{S}$ generally can not be equivalently shown as $\mathbf{M}=\mathbf{R}^{\prime} \mathbf{D}$. So, we can conclude that it represents a larger set for $\mathbf{M}$ by using a symmetric B. Fig. 3 shows an example of this difference.

\subsection{Effect on Transparency}

As mentioned before, transparency of a teleoperation system is the equality between impedance of the environment $Z_{e}$ and the displayed impedance to the human side $Z_{h}$. In other words, transparency is achieved if

$$
Z_{h}(s)=Z_{e}(s),
$$

where $s$ is a complex Laplace domain variable [2]. By neglecting the controller and teleoperator robot's dynamics, displayed impedance in the human side $Z_{h}$ is calculated based on the environment impedance $Z_{e}$ and generalized scattering transformation

$$
Z_{h}=\frac{\mu_{21}-\mu_{11} \Phi e^{-s T}}{-\mu_{22}+\mu_{12} \Phi e^{-s T}}, \Phi=\frac{\mu_{21}+\mu_{22} Z_{e}}{\mu_{11}+\mu_{12} Z_{e}},
$$

where $T=T_{1}+T_{2}$ the round trip time delay and

$$
\mathbf{M}=\left[\begin{array}{ll}
\mu_{11} & \mu_{12} \\
\mu_{21} & \mu_{22}
\end{array}\right] \text {. }
$$

In [10], a low frequency analysis is given which extracts the displayed impedance assuming a diagonal $\mathrm{B}$ and Padé approximation for time delay for $\omega<\frac{1}{3 T}$.

Here we calculate $Z_{h}$ without any approximations by rewriting the equation (7) without restricting the $Z_{e}$ to low frequencies. Then we have

$$
\begin{aligned}
Z_{h} & =\frac{\mu_{11} \mu_{21}+Z_{e} \mu_{12} \mu_{21}-\mu_{11} \mu_{21} \mathrm{e}^{-T s}-Z_{e} \mu_{11} \mu_{22} \mathrm{e}^{-T s}}{\mu_{12} \mu_{21} \mathrm{e}^{-T s}-Z_{e} \mu_{12} \mu_{22}-\mu_{11} \mu_{22}+Z_{e} \mu_{12} \mu_{22} \mathrm{e}^{-T s}} \\
& =\frac{Z_{e}\left(\mu_{12} \mu_{21}-\mu_{11} \mu_{22} e^{-T s}\right)+\mu_{11} \mu_{21}\left(1-e^{-T s}\right)}{\left(\mu_{12} \mu_{21} e^{-T s}-\mu_{11} \mu_{22}\right)+Z_{e} \mu_{22}\left(\mu_{12} e^{-T s}-\mu_{12}\right)} .
\end{aligned}
$$

Looking into the equation above, it can be seen that to have $Z_{h} \approx$ $Z_{e}$ we must have large values for $\mu_{11} \mu_{22} \gg \mu_{12} \mu_{21}$. This means for an optimal design we should have $\operatorname{Det}(M) \gg 0$ and also $\mu_{21}$ and $\mu_{22}$ should be small values.

\subsection{Effect on Disturbance rejection}

As shown in Fig.4, the transfer functions of the right- and left-hand side systems, $Z_{r}$ and $Z_{l}$, seen from the communication loop, respectively can be calculated as

$$
\begin{aligned}
& Z_{r}=\frac{v_{r}}{u_{r}}=\frac{\mu_{21}+\mu_{22} Z_{e}}{\mu_{11}+\mu_{12} Z_{e}}, \\
& Z_{l}=\frac{u_{l}}{v_{l}}=\frac{\mu_{12}-\mu_{11} Z_{m}}{\mu_{22}-\mu_{21} Z_{m}} .
\end{aligned}
$$

Combining the above two transfer functions with the assumption of the received variable on the teleoperator side is disturbed, i.e. $u_{r}=u_{l}+d$, the force feedback disturbance rejection transfer function is written as

$$
f_{f} \text {-disturbance rejection }=\frac{f_{f}}{d}=\frac{1}{\mu_{22}+\mu_{21} Z_{m}} \cdot \frac{Z_{r}}{1-Z_{r} Z_{l}} .
$$

Substituting the equations 10 and 9 into equation 11 gives the following equation

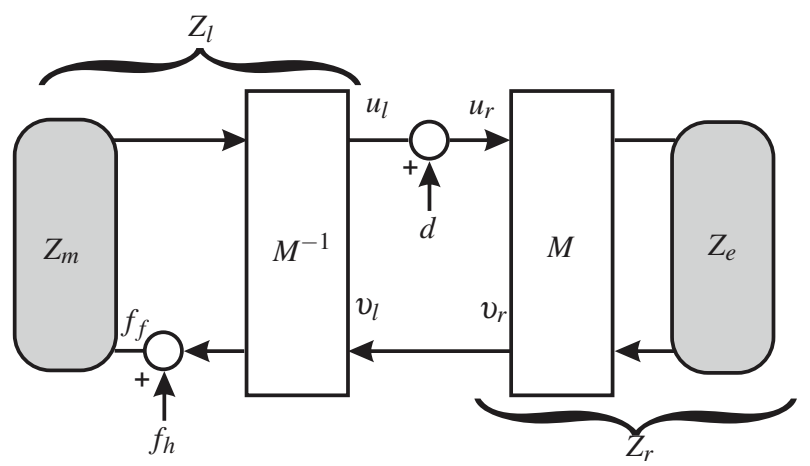

Figure 4: Disturbance signal $d$ affects the signal in communication channel. $Z_{e}$ the environment's impedance and $Z_{m}$ in the human and HSI impedance. 


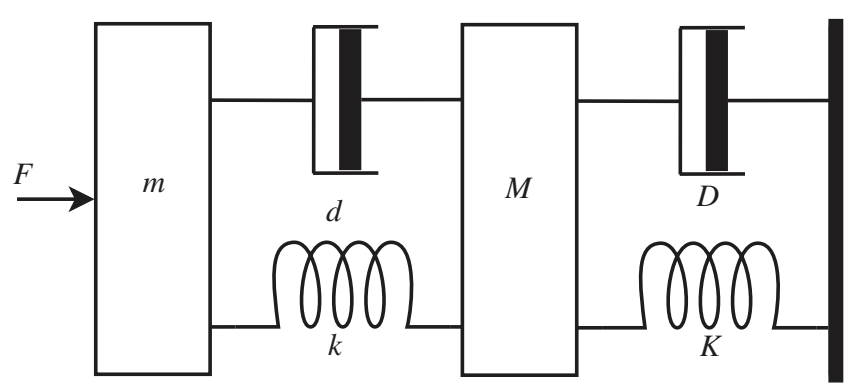

Figure 5: Double circuit model of the human eye

$$
\begin{aligned}
\frac{f_{f}}{d} & =\frac{\left(\mu_{22}-Z_{m} \mu_{21}\right) \cdot\left(\mu_{21}+Z_{s} \mu_{22}\right)}{\left(Z_{m} Z_{s}+1\right) \cdot\left(\mu_{11} \mu_{22}-\mu_{12} \mu_{21}\right) \cdot\left(\mu_{22}+Z_{m} \mu_{21}\right)} \\
& =\frac{\left(\mu_{22}-Z_{m} \mu_{21}\right) \cdot\left(\mu_{21}+Z_{s} \mu_{22}\right)}{\left(Z_{m} Z_{s}+1\right) \cdot \operatorname{Det}(M) \cdot\left(\mu_{22}+Z_{m} \mu_{21}\right)} .
\end{aligned}
$$

Practically, we are interested on the maximum attenuation of the disturbance. As it is seen in the transfer function, maximum attenuation is achieved when the same properties for matrix $\mathrm{M}$ is used which is large determinant value for $\operatorname{Det}(M) \gg 0$ and small values for $\mu_{21}$.

\section{QuANTITATIVE TRANSPARENCY MEASURE}

In this work, for sake of performance analysis of our approach, we will use double-circuit model of the human eyeball as shown in Fig.5 in order to compare according to existing and conventional transparency measures. The model is consisting of a low-frequency part which is related to the under laying tissue of the eyeball, and the high-frequency part which is originated from indenter tissues [16]. Velocity to force mapping by environment is described by the following frequency response model:

$$
G(s)=\frac{F(s)}{V(s)}=\frac{1}{m S+\left(\frac{D K S}{D K M S^{2}+K S+D}+\frac{d k S}{d+k S}\right)^{-1}}
$$

Where $K, D$ and $M$ are low frequency parameters, and $k, d$ and $m$ are high frequency resonance parts respectively. Non of the low frequency parameters change noticeably by the indenter type or the force [16] but high frequency parameter $m$ can range depending the diameter of the indenter. The effect of this range is shown in the Fig.6 for range of $10 \%$. Thus, to avoid precise parameter identification requirement we use only dissipativity parameters of systems which is described in the following.

For the maximum transparency, we must find four variables of $b_{11}, b_{12}, b_{21}, b_{22}$ in $\mathbf{B}$ which minimizes the difference between $Z_{h}$ in equation (8) and the environment impedance $Z_{e}$. As a measure for this minimization, we use error vector magnitude (EVM) proposed

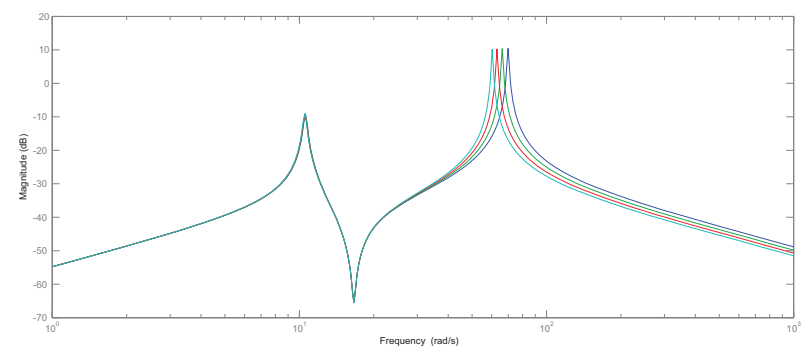

Figure 6: Magnitude-frequency diagram of the model for $10 \%$ range of $m$ in [17] with slight changes. For the best similarity between $Z_{h}$ and $Z_{e}$, we should have a small $H_{2}$ norm for $\Delta Z(s)=Z_{h}(s)-Z_{e}(s)$ which gives

$$
J_{m}=\|\Delta Z(j \omega)\|_{2}^{2}=\int_{-\omega_{0}}^{\omega_{0}} \Delta Z(j \omega) \Delta Z(j \omega)^{*} d \omega,
$$

where $J_{m}$ is the defined cost for a given frequency range of $\omega_{0}$ and $m$. Hence the design problem is reduced to minimization of summation of these differences,

$$
J=\sum_{m_{\min }}^{m_{\max }} J_{m}
$$

where $m_{\min }$ and $m_{\max }$ are the boundary values for $m$. Number of grid points for $m$ can be arbitrarily defined. Finally our used optimization problem can be written as,

$$
\begin{aligned}
& \underset{b_{i j}}{\arg \min _{i} J} \\
& \text { subject to: } \\
& b_{11} \cdot b_{22}-b_{12} \cdot b_{21} \neq 0 \\
& 0 \leq b_{i j} \leq b_{\text {max }}
\end{aligned}
$$

where $i, j \in\{1,2\}$ and $b_{\max }$ is the maximum permissible value for every $b_{i j}$. Since the required properties of $\mathbf{M}$ (which are extracted and presented in section 4) are more of qualitative measures than explicit values, we use PSO technique to search for local or possible global minimums in (15). PSO method is inspired by social behaviors of moving birds flock, where birds (particles) are moving in a space in order to achieve a common goal like finding food. For extensive studies, reader can refer to the resources like [18] and [19].

\section{Simulations Results}

First, we will examine the performance of the algorithm by a numerical example on a simple environment: we assume the environment is a time invariant spring-damper with $Z_{e}=\frac{500}{s}+20$. Since the dissipativity parameters $\sigma_{e}=20$ and $\varepsilon_{e}=0$, then the dissipativity matrix of environment $\mathbf{P}_{e}$ is an IFP system and will be

$$
\mathbf{P}_{e}=\left[\begin{array}{cc}
-20 & \frac{1}{2} \\
\frac{1}{2} & 0
\end{array}\right] \text {. }
$$

The left hand side system is assumed as any system with dissipativity parameters which satisfies the finite gain $\mathscr{L}_{2}$-stability conditions in proposition 1 . Here, we assume an OFP system $\Sigma_{l}$, with $\sigma_{l}=0$ and $\varepsilon_{l}=10$. According to proposition 1, the feedback interconnection of these systems without considering the time delay is stable since

$$
\varepsilon_{e}+\sigma_{l}>0, \varepsilon_{l}+\sigma_{e}>0 .
$$

To avoid local minimums due to use of swarm optimization, simulations are repeated 10 times for $\omega_{0}=100$. The smallest given cost function value which was around 2900, was repeated in each run of the simulation. So we assume that this cost value and it's suggested parameters are the best we can get from this method of optimization and given frequency points.

Table 1: Suggested optimal values for $b_{i j}$

\begin{tabular}{ccccc} 
Element & $b_{11}$ & $b_{12}$ & $b_{21}$ & $b_{22}$ \\
\hline Value & 76.51 & -2.8 & -2.8 & 4.28
\end{tabular}




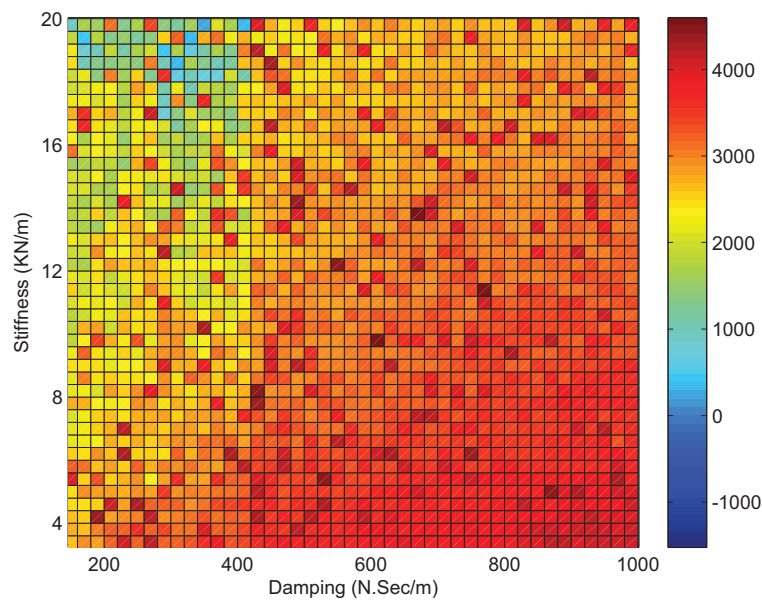

Figure 7: Difference heat map between diagonal and symmetric B cost function.

The calculated value for the $\mathbf{B}$ has been shown in table 1. By having the elements of $\mathbf{B}$, we can calculate the stabilizing rotation angle for $\mathbf{M}$ by using (5) and (4). So according to (5) $\mathbf{P}_{B}$ is calculated as

$$
\mathbf{P}_{B}=\left[\begin{array}{cc}
-0.0035 & -0.0007 \\
-0.0007 & 0.0006
\end{array}\right]
$$

and the stabilizing rotation angle is achieved from inversion of (4) gives $\theta=44.50^{\circ}$. By having $\theta$, and calculating the matrix $\mathbf{M}$ according to (3), appeared dissipativity matrix for $\Sigma_{3}$ is equal to the environment which means $\mathbf{P}_{3}=\mathbf{P}_{e}$. Now the designed system is stable since the dissipativity values of the system is appeared as the same to the left side.

In the next step, for an extensive and overall comparison of the designed system performance, a very large set of the systems are simulated by the given model of

$$
G(S)=\frac{V(s)}{F(s)}=\frac{s}{m s^{2}+b s+k},
$$

where $m$ is the inertia which is assumed to be a fixed value $m=1$, $b$ is damping and $k$ is the stiffness coefficient of the environment model. Simulations are performed by sliding the parameters $b$ and $k$ in the range of $100<b<2000\left(\frac{N . s}{m}\right)$ and $2<k<20\left(\frac{k N}{m}\right)$ with resolution of 50 steps for each coefficient. The system is positive real for every positive values of $m, b$ and $k$. As a result, the requirement of being passive for the environment is also satisfied. As mentioned before, the inertia of the system is fixed as $1 \mathrm{Kg}$, damping and stiffness coefficients are changed in 50 steps. In total 2025 different systems from a same class of mass-spring-damper are simulated in both diagonal and symmetric B. Dissipativity values of the systems are calculated by CVX toolbox [20] [21]. Fig.7 shows the 2D heat map of difference between two set of cost functions. The value on each pixel of the map in assigned by

$$
D_{i j}=J_{\text {diag }}^{*}\left(b_{i j}, k_{i j}\right)-J_{\text {sym }}^{*}\left(b_{i j}, k_{i j}\right) .
$$

As it can be seen in the Fig.7, cost function for set of symmetric $\mathbf{B}$ is always lower and therefore $D_{i j}$ is always positive. However some noisy pixels (cost function values) are seen on the map which is due to the nature of randomized optimization. Fig.8, shows the relative ratio between $J_{d i a g}^{*}\left(b_{i j}, k_{i j}\right)$ and $J_{s y m}^{*}\left(b_{i j}, k_{i j}\right)$ which is calculated by

$$
R_{i j}=\frac{J_{\text {diag }}^{*}\left(b_{i j}, k_{i j}\right)}{J_{s y m}^{*}\left(b_{i j}, k_{i j}\right)} .
$$

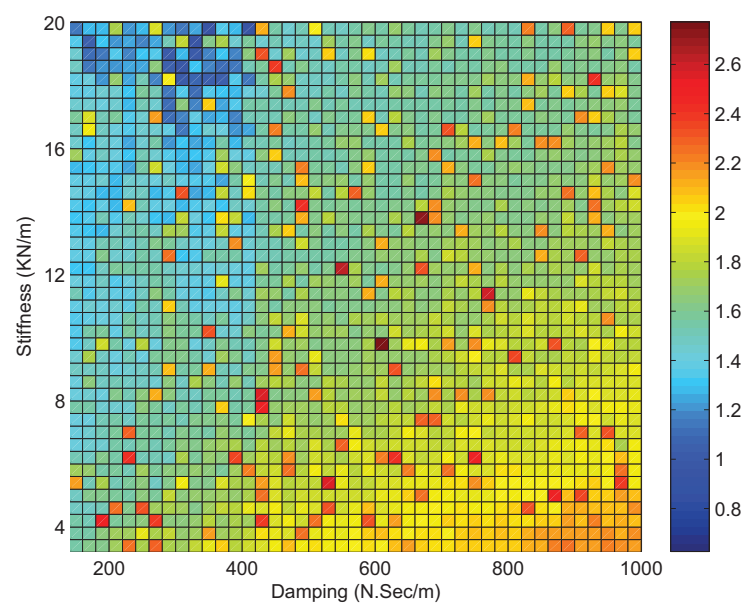

Figure 8: Ratio heat map of diagonal and symmetric B cost function

The $R_{i j}$ is also always larger than one, and the interesting interpretation from the figure is, the faster the dynamics of the system becomes (more stiffness), the larger becomes $R_{i j}$. It means symmetrical $\mathbf{B}$ provides higher transparency in the systems with higher impedance, meanwhile performing better or equal to the set of diagonal $\mathbf{B}$ in slower systems.

\section{EXPERIMENTAL RESULTS}

Real experiments are also done for comparing the achieved values from optimizations on a teleoperation robot. By using impedance Cartesian control and using high gain motion controllers, without loss of generality, we can extend the result of a 1-DOF cartesian robot to mutli-DOF robots. So, we use a haptic interface consists of a 1 DOF linear-actuated with an end-effector at the grasping point for the user. A (JR3) force/torque sensor measures the force that human feels at the grasping point. Human and 1 DOF actuator, act as the Human-HSI part of the system and environment is virtually made by a software impedance model. Velocity of the human hand is measured by the linear actuator's encoder and sent to the virtual model. Motion controller is a very high gain PD controller, so dynamics of the robot is neglected. The force which is generated by the model is sent back to the HSI to be applied on human with the sampling rate of $1 \mathrm{khz}$. Constant $10 \mathrm{~ms}$ time delay is placed in both signal forward and backward paths. In Fig. 9, configuration of the human and HSI device is depicted. All software parts are implemented in MATLAB/Simulink on the Linux/PreemptRT using MATLABs Real-Time Workshop.

\subsection{Comparision of transparency}

Virtual environment is assumed to be the model given in (13) with the following parameters according to experiments in [16]:

\begin{tabular}{|l|c|c|}
\hline$m=0.278 g$ & $k=1312 N / m$ & $d=0.483 N s / m$ \\
$M=8.2 g$ & $K=951 N / m$ & $D=3.03 N s / m$ \\
\hline
\end{tabular}

Optimizations are done based on (14) for a $\pm 5 \%$ percent (total $10 \%$ ) of variation for the given $m$ for both symmetric and diagonal B. Since the used device for the experiments was suitable only for large forces and displacements, velocity/force signal has been properly scaled to make it perceivable for the operator. To keep the loop gain the same, scaling values for force and velocity has been chosen inverse of each other. Human position and reflected force is recorded for around 10 seconds with sample rate of $1 \frac{K s}{\sec }$. A polynomial regression has been applied on around 10000 data samples by Least Square algorithm to estimate the impedance values that appears for human on the HSI. 


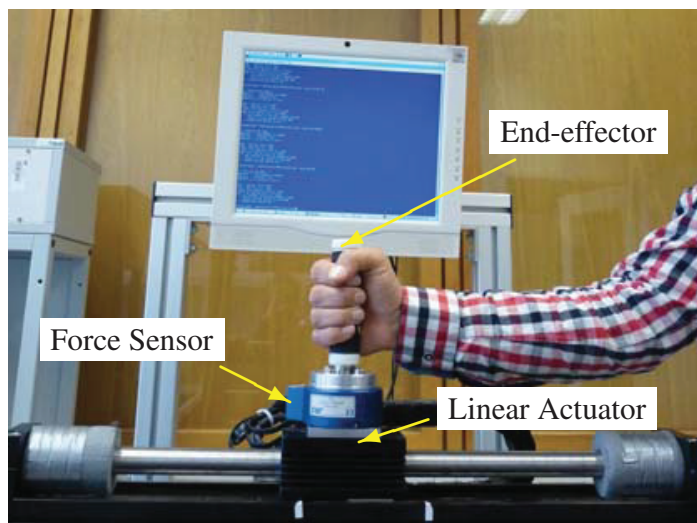

Figure 9: Configuration of Human, 1 DOF HSI and the connected force sensor.

\begin{tabular}{|c|c|c|c|}
\hline Element & Original & Diagonal $B$ & Symmetric $B$ \\
\hline $\bar{M}$ & 8.2 & 10.87 & 9.24 \\
\hline$K$ & 951 & 313 & 625 \\
\hline$D$ & 3.03 & 4.39 & 4.01 \\
\hline$m$ & 0.27 & .63 & 0.51 \\
\hline$k$ & 1312 & 474 & 407 \\
\hline$d$ & 0.483 & 0.85 & 0.88 \\
\hline
\end{tabular}

Table 2 and 3 shows the comparison between performance of each kind of $\mathbf{B}$ matrix. Confirming the simulation results, Symmetric matrix acts better in transparency. For low frequency part mass is almost perfectly rendered, stiffness and damping are still better than diagonal case. For high frequency part both did not show very good performance which can be because of wave filtering in the communication channel.

\section{Conclusions}

In this work we applied broader class of parameters to design the generalized scattering parameters. Furthermore, some necessary requirements on transformation matrix are extracted which should be satisfied by any design method. Then, we scaled down this requirements, to an optimization problem with a cost function based on error vector transfer function's magnitude. Although we used a randomized method for the optimization, simulations are repeated several times to ensure the final answer is not trapped in a local minimum. For each given damping and stiffness values, the best result of these optimization are chosen. Simulations showed a major improvement in displayed impedance in its lower range of frequency which is impornant in order to apply excessive force on the tissue. Using a symmetric matrix instead of diagonal form for matrix $\mathbf{B}$ gives more freedom to satisfy the requirements for matrix $\mathbf{M}$ given in section 4. Experiments also confirmed the simulation results and the proposed changes in the matrix $\mathbf{B}$. A constructive design method for $\mathbf{B}$ is still missing and considered as a future work. By using a more comprehensive cost function, which considers the desired transparency and stability margins together, we can adjust the performance of the system depending on the situations.

\section{REFERENCES}

[1] M.J. Massimino and T.B. Sheridan. Teleoperator performance with varying force and visual feedback. Human Factors: The Journal of the Human Factors and Ergonomics Society, 36(1):145-157, 1994.
Table 3: Rendering error in \%

\begin{tabular}{ccc} 
Element & Diagonal $B$ & Symmetric $B$ \\
\hline$M$ & $32 \%$ & $12 \%$ \\
$K$ & $67 \%$ & $34 \%$ \\
$D$ & $44 \%$ & $32 \%$ \\
$m$ & $133 \%$ & $88 \%$ \\
$d$ & $63 \%$ & $68 \%$ \\
$k$ & $94 \%$ & $101 \%$
\end{tabular}

[2] D.A. Lawrence. Stability and transparency in bilateral teleoperation. Robotics and Automation, IEEE Transactions on, 9(5):624-637, 1993.

[3] T.B. Sheridan. Space teleoperation through time delay: Review and prognosis. Robotics and Automation, IEEE Transactions on, 9(5):592-606, 1993

[4] G. Niemeyer and J.J.E. Slotine. Stable adaptive teleoperation. Oceanic Engineering, IEEE Journal of, 16(1):152-162, 1991.

[5] R.J. Anderson and M.W. Spong. Bilateral control of teleoperators with time delay. Automatic Control, IEEE Transactions on, 34(5):494-501, 1989.

[6] S. Hirche, M. Ferre, J. Barrio, C. Melchiorri, and M. Buss. Bilateral control architectures for telerobotics. Advances in Telerobotics, pages 163-176, 2007.

[7] N. Diolaiti, C. Melchiorri, and S. Stramigioli. Contact impedance estimation for robotic systems. Robotics, IEEE Transactions on, 21(5):925-935, 2005.

[8] S. Hirche, T. Matiakis, and M. Buss. A distributed controller approach for delay-independent stability of networked control systems. Automatica, 45(8):1828-1836, 2009.

[9] T. Matiakis, S. Hirche, and M. Buss. Independent-of-delay stability of nonlinear networked control systems by scattering transformation. American Control Conference, 2006, pages 6-12, 2006.

[10] I. Vittorias and S. Hirche. Stable teleoperation with communication unreliabilities and approximate human/environment dynamics knowledge. American Control Conference (ACC), 2010, pages 2791-2796, 2010.

[11] M.A. Nasseri, M. Eder, D. Eberts, S. Nair, M. Maier, D. Zapp, C.P. Lohmann, and A. Knoll. Kinematics and dynamics analysis of a hybrid parallel-serial micromanipulator designed for biomedical applications. In Advanced Intelligent Mechatronics (AIM), 2013 IEEE/ASME International Conference on, pages 293-299, 2013.

[12] P. Kokotović and M. Arcak. Constructive nonlinear control: a historical perspective. Automatica, 37(5):637-662, 2001.

[13] I. Vittorias and S. Hirche. Transparency of the generalized scattering transformation for haptic telepresence. Haptics: Generating and Perceiving Tangible Sensations, pages 183-188, 2010.

[14] H.K. Khalil. Nonlinear systems. Prentice Hall PTR, 2002.

[15] J.E. Marsden and T.J.R. Hughes. Mathematical foundations of elasticity. Dover Publications, 1994.

[16] EM Timanin and EV Eremin. Registration and modeling of stiffness and impedance of a human eye.

[17] J. Kim and P.H. Chang. Extended transparency as a quantitative performance measure in bilateral teleoperation. Intelligent Robots and Systems, 2007. IROS 2007. IEEE/RSJ International Conference on, pages 3119-3124, 2007.

[18] X.S. Yang. Nature-inspired metaheuristic algorithms. Luniver Press, 2011.

[19] M. Clerc. Discrete particle swarm optimization illustrated by the traveling salesman problem. New optimization techniques in engineering, 141:219-239, 2004.

[20] M. Grant and S. Boyd. Cvx: Matlab software for disciplined convex programming, v. 2, 2012.

[21] M. Grant and S. Boyd. Graph implementations for nonsmooth convex programs. Recent advances in learning and control, pages 95-110, 2008. 